\title{
Comparison of the vertical bone defect healing abilities of carbonate apatite, $\beta$-tricalcium phosphate, hydroxyapatite and bovine-derived heterogeneous bone
}

\author{
Nobuya SATO, Keisuke HANDA, Venkata Suresh VENKATAIAH, Tatsuya HASEGAWA, Mary M. NJUGUNA, \\ Yoshio YAHATA and Masahiro SAITO
}

Department of Restorative Dentistry, Division of Operative Dentistry, Tohoku University Graduate School of Dentistry, 4-1 Seiryo-machi, Aoba-ku, Sendai, Miyagi 980-8575, Japan

Corresponding author, Keisuke HANDA; E-mail: khanda@dent.tohoku.ac.jp

\begin{abstract}
The treatment of vertical bone defects caused by severe periodontal disease requires the regeneration of periodontal tissue. Although various bone substitutes have been clinically applied to vertical bone defect correction, the evaluation of these materials in periodontal tissue remains incomplete. The purpose of this study was to examine the bone regeneration abilities of various bone substitutes including Cytrans, Cerasorb, Neobone and Bio-Oss in a 3-wall bone defect animal model. All of these bone substitutes showed a similar healing ability to periodontal ligament and cementum. However, Cytrans showed the fastest bone healing ability compared with the other materials at 4 weeks post-transplantation. In addition, the recruitment of osteoclasts and endothelial cells was observed in Cytrans grafts at 4 weeks, but only detected at 8 weeks in the other materials. These results suggest that Cytrans promotes faster bone healing by inducing bone remodeling and angiogenesis.
\end{abstract}

Keywords: Carbonate apatite, Bone substitutes, Bone regeneration, Angiogenesis

\section{INTRODUCTION}

Periodontal tissue consists of strong connective tissue mainly composed of type I collagen that can withstand occlusal force. This tissue undergoes irreversible destruction however as a result of periodontal pathogeninduced chronic inflammation. Periodontitis is a chronic inflammatory disease of connective tissue caused by bacterial plaque adhering to the cervical part of this tissue. The WHO has reported that more than $20 \%$ of the world's population suffers from periodontitis, making it the most prominent infectious disease in humans. It is vital therefore to develop regenerative medicine applications to treat highly degraded periodontal tissue to preserve teeth. Vertical bone defects are a diagnostic phenomenon of severe periodontitis and require surgical therapy. However, bone replacement is necessary in these cases as spontaneous recovery of a vertical bone defect is limited. The ideal material for bone replacement needs to be biocompatible, bioresorbable, osteoconductive, be structurally similar to bone, have adequate mechanical strength, be safe to handle, and be cost effective. To satisfy these requirements, bone grafting has been developed with synthetic materials.

Bone grafting materials are currently classified as of biological origin (autogenous bone, allogeneic bone, and heterogeneous bone) and artificial bone (hydroxyapatite $[\mathrm{HA}], \beta$-tricalcium phosphate $[\beta-\mathrm{TCP}]$, bioactive glass, and calcium sulfate). Autologous bone has become the gold-standard in bone grafting material as it contains

Color figures can be viewed in the online issue, which is available at J-STAGE.

Received Mar 25, 2019: Accepted Apr 25, 2019

doi:10.4012/dmj.2019-084 JOI JST.JSTAGE/dmj/2019-084 growth factors in its matrix that help to regenerate bone defects. Freeze-dried bones and demineralized freeze-dried bones collected from human cadavers can work well for new bone formation, but carry immunological and ethical issues, as well as a risk of viral infection. Bovine-derived heterogeneous bone is composed of carbonate-containing apatite with the same main component as natural bone and thus has high biocompatibility, but bone healing with this material is dependent on the experimental conditions ${ }^{1-4)}$. In recent years, the development of synthetic bone substitutes with osteoinductive ability has been promoted to compensate for the limitations of using autologous bone. These materials are classified as TCP or HA. Although HA has osteoconductive ability and excellent biocompatibility, it requires a long time to replace natural bone. In contrast, $\beta$-TCP has higher solubility in physiological saline than $\mathrm{HA}$, as well as better bone conductivity and bioresorbability ${ }^{5}$. Many bone substitutes including $\beta$-TCP have already been clinically applied $^{6}$, but further development of bone substitute materials that can produce faster and more reliable bone healing is anticipated.

Compared with HA and $\beta$-TCP, carbonate apatite $\left(\mathrm{CO}_{3} \mathrm{Ap}\right)$ can also be easily substituted for bone because its hydroxyl group is replaced with a carbonate group, and it therefore produces a skeleton has a porous microstructure that is morphologically similar to that of human cancellous bone ${ }^{7}$. $\mathrm{CO}_{3} \mathrm{Ap}$ granules show greater bone formation in the cortical portion of a bone defect than Bio-Oss, which is a deproteinized bovine bone mineral ${ }^{8}$. $\mathrm{CO}_{3} \mathrm{Ap}$ has also shown faster bone healing 
than HA via the activation of bone metabolism, which stimulates bone matrix formation by osteoblasts and bone absorption by osteoclasts ${ }^{9)}$. Clinical studies using $\mathrm{CO}_{3} \mathrm{Ap}$ for maxillary sinus surgery and immediate implantation have indicated that bone resorption does not occur at the $\mathrm{CO}_{3} \mathrm{Ap}$ implantation site, that bone height is maintained, and that no failure or complication of the implant including abnormal bone resorption is observed until one year later, indicating that $\mathrm{CO}_{3} \mathrm{Ap}$ is a suitable bone substitute material for bone healing ${ }^{10)}$.

Although the effectiveness of bone healing has been demonstrated in animal bone defect models and in maxillary sinus surgery, little research has been conducted on vertical bone defect healing. To address this issue, a 3 -wall bone defect dog model was created in our current study to evaluate the efficacy of $\mathrm{CO}_{3} \mathrm{Ap}$ and compare its vertical bone defect healing abilities with those of Bio-Oss, HA and $\beta$-TCP.

\section{MATERIAL AND METHODS}

\section{Experimental animals}

Sixteen healthy adult male beagles aged 12 months and with fully erupted permanent dentition were used in this study. The animal selection, management, surgical protocols and preparation procedures were approved by the animal experiment ethics committee of HAMRI (approval No-16-H058). The following bone substitutes were investigated: Bio-Oss ${ }^{\circledR}$ (deproteinized bovine bone material; Geistlich Pharma, Wolhusen, Switzerland), Cytrans $^{\circledR} \quad\left(\mathrm{CO}_{3} \mathrm{Ap}\right.$, carbonate apatite; GC, Tokyo, Japan), Cerasorb ${ }^{\circledR}$ ( $\beta$-TCP, $\beta$-tri-calcium phosphate; Curasan, Frankfurt Germany), and Neobone ${ }^{\circledR}$ (HA, hydroxyapatite; CoorsTec, Tokyo, Japan). Control material was also used in a sham operated group. The 16 animals were randomly divided into 2 groups of 8 animals in accordance with the experimental timepoint, i.e., 4 or 8 weeks post-transplantation. Each animal received different combinations of bone substitute materials on either side of the jaw with a sample size of three for each material under investigation. All surgical procedures were performed under general anesthesia with ketamine hydrochloride and xylazine. As a baseline, two mandibular premolars on both the left and right sides of the mandible were extracted, and extraction sockets were allowed to heal for 8 weeks (Fig. 1a). Defects were then prepared in the premolar region (site of previous extraction) on both the left and right jaw with dimensions of a $4 \mathrm{~mm}$ width (bucco-lingual), $5 \mathrm{~mm}$ depth (apico-coronal) and $5 \mathrm{~mm}$ length (mesio-distal)

(a)

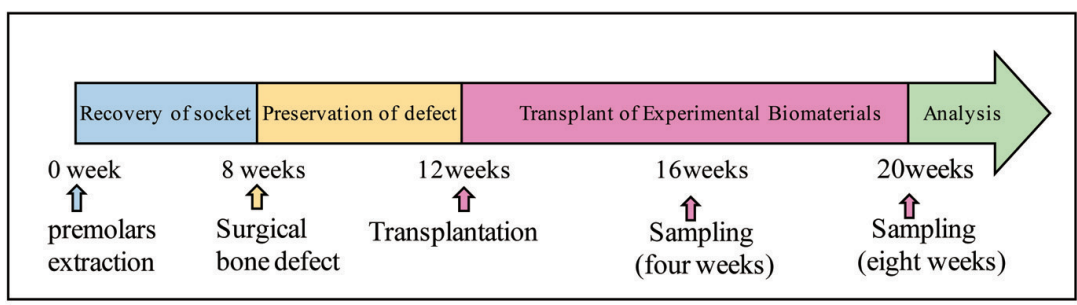

(b) Buccal view

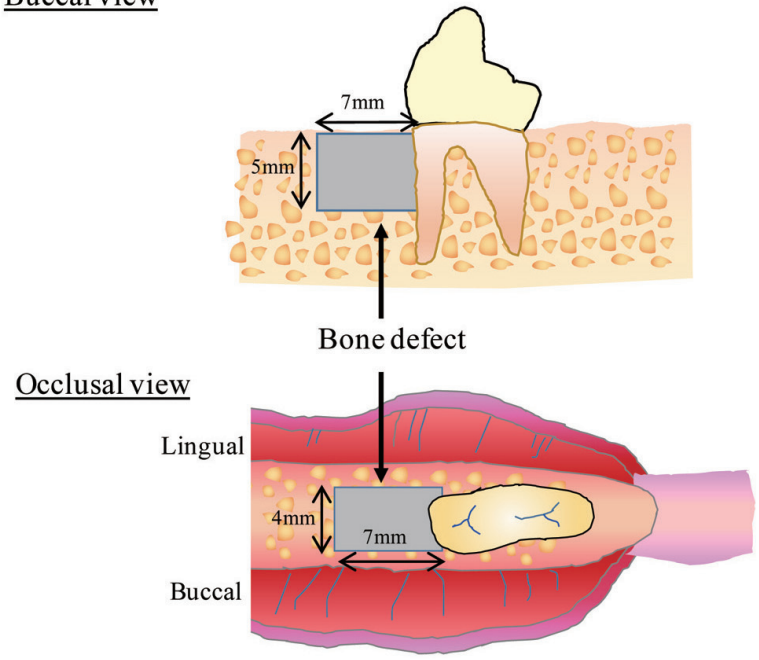

Fig. 1 (a) Schematic illustration of the timeline of this study. (b) Schema for the establishment of bone defects in the animal model, shown in the in bucco-lingual and occlusal view. Arrows indicate the position of the bone defect. 
(Fig. 1b).

The defects were created using the sterile carbide burs in a low-speed handpiece under continuous sterile saline irrigation. Standardization of all defects was achieved using an acrylic template that had the above dimensions. The defect was then immediately filled with the bone substitute, which had been cleaned using saline, and the flaps were closed and sutured to allow for full coverage of bone substitutes within the defect. The animals were euthanized at 4 or 8 weeks ostoperatively, and the mandibles were then dissected.

\section{Microcomputed tomography analysis}

Bone regeneration was examined by microcomputed tomography $(\mu \mathrm{CT})$ scanning. Three-dimensional $\mu \mathrm{CT}$ images were analyzed and quantified using image analysis software (TRI/3D-BON; Ratoc System Engineering, Tokyo, Japan).

\section{Bone-morphometric analysis}

Alveolar bone loss was analyzed by measuring the distance from the lingual cement-enamel junction (CEJ) of the second molar to the lingual-mesial root to the lingual alveolar bone crest (ABC) parallel to the sagittal plane. The distance from the CEJ to the ABC (ABC-CEJ) was used to quantify bone regeneration.

\section{Histological analysis}

Following the $\mu \mathrm{CT}$ analysis, the tissues were decalcified in a solution containing $50 \%$ formic acid and $20 \%$ citric acid. The samples were then dehydrated through a graded series of ethanol, cleared with xylene and embedded in paraffin. Serial sections $(5 \mu \mathrm{m})$ were cut in the mesio-distal plane throughout the buccal-lingual extension of the teeth. The sections were deparaffinized and stained with hematoxylin-eosin (HE) to evaluate the formation of new bone and Azan stained to evaluate the formation of collagen fibers. Sections were then observed under a light microscope (DM6000B, Leica Microsystems, Wetzlar, Germany) fitted with a digital video camera. Photographs were taken of the entire defect at a $2.5 \times$ magnification, and of the periodontal area at a $40 \times$ magnification. The new bone area in the defect was assessed using ImageJ software (US National Institutes of Health, Bethesda, MD, USA).

\section{Immunohistochemical analysis}

Immunohistochemical analysis was done to evaluate neo-vascularization by checking for CD31-positive cells and to determine resorption of bone by checking for cathepsin K, an osteoclast marker. The formalinfixed, paraffin-embedded specimens were cut into $4-\mu \mathrm{m}$ thick sections and placed on glue-coated glass slides for immunohistochemistry. Briefly, the samples were deparaffinized in xylene and hydrated in a graded alcohol series and distilled water. Endogenous peroxidase activity was blocked with $3 \%$ hydrogen peroxidase for $10 \mathrm{~min}$. The samples were then incubated for $30 \mathrm{~min}$ at room temperature in a blocking solution with $1 \%$ rabbit serum (Nichirei Bioscience, Tokyo, Japan). An anti-CD31 polyclonal antibody (Funakoshi, Tokyo, Japan) reaction was then performed for $16 \mathrm{~h}$ at $4^{\circ} \mathrm{C}$. Binding of the first antibody was visualized using a Histofine kit (Nichirei Bioscience) with DAB (3,3-diaminobenzidine). The sections were then examined using a Leica DM6000B microscope (Leica Microsystems) fitted with a digital video camera (Leica MC 170HD, Leica Microsystems).

\section{Statistical analysis}

The results were analyzed and collected using GraphPad Prism 6 software (La Jolla, CA, USA) and samples were tested in triplicate. The means and standard deviations were calculated and the statistical significance of the differences between groups was examined using one-way analysis of variance (ANOVA) followed by a Dunnett's multiple comparison test. Test results were considered significant at probability $p$ values $<0.05$.

\section{RESULTS}

All of the animals survived for the entire experimental period without any complications. Bone defect sites grafted with various bone-regenerating biomaterials were microscopically homogenous, with clear, soft tissue healing covering the defect area. There were no clinical signs of inflammation or infection at the experimental site at either 4 or 8 weeks after grafting.

\section{Micro-CT evaluation of in vivo bone regeneration ability of grafted biomaterials.}

The $\mu \mathrm{CT}$ images of the defect areas grafted with different biomaterials acquired after 4 and 8 weeks are shown in Figs. 2 and 3. We established a dog bone defect model to investigate the bone regeneration ability of the biomaterials Cytrans, Bio-Oss, Cerasorb, and Neobone and compare them to a sham (control) group. Threedimensional (3D) reconstruction of $\mu \mathrm{CT}$ images of the grafted areas revealed new alveolar bone formation in all four experimental groups. Under this $3 \mathrm{D}$ view, the yellow area inside the defect outlined by the arrowheads represents the newly formed alveolar bone. In the coronal, axial and sagittal views of the control group, we observed the persistence of radiolucency in the defect area (outlined by an arrowhead) at 4 and 8 weeks, suggesting minimal bone formation (Figs. 2 and 3). A variable amount of radiopacity (outlined by arrowheads) was observed in the coronal, axial and sagittal views in all of the experimental groups at 4 and 8 weeks, indicating new bone formation. The amount of this new bone formation in each group was further evaluated by quantitative $\mu \mathrm{CT}$ analysis.

Although there was higher new bone volume percentage in the Cytrans group at 4 weeks after transplantation compared to Bio-Oss, Cerasorb and Neobone, there were no significant differences between these three groups (Fig. 4a). Bone volume analysis confirmed that the Cytrans group was significantly higher, at $30 \%$ over the sham group and $28 \%$ over the Bio-Oss group at 4 weeks (Fig. 4 a). In contrast, at 8 weeks post-transplantation, there were no significant 


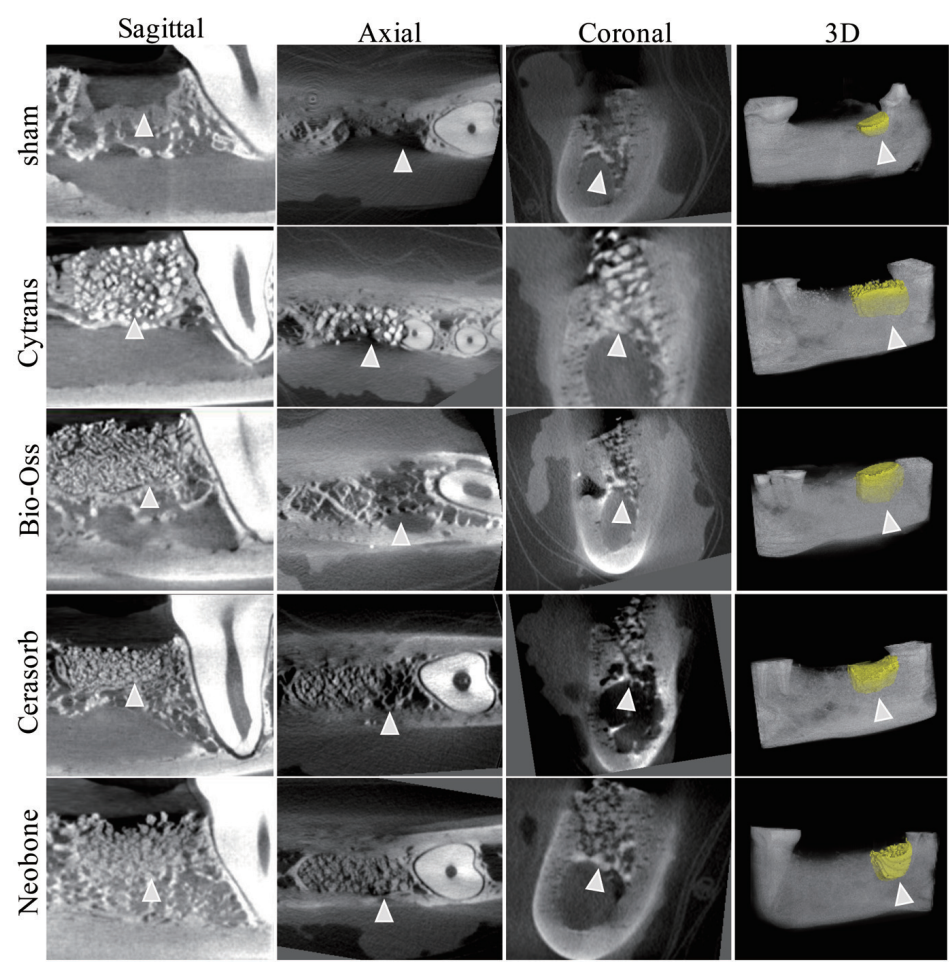

Fig. 2 Micro-CT analysis of new bone regeneration after 4 weeks of healing period in the animal model. Axial, sagittal, coronal and reconstructed 3D microcomputed tomography (CT) images of the defect sites in the control group (sham) and the Cytrans, Bio-Oss, Cerasorb and Neobone groups at 4 weeks post-transplantation.

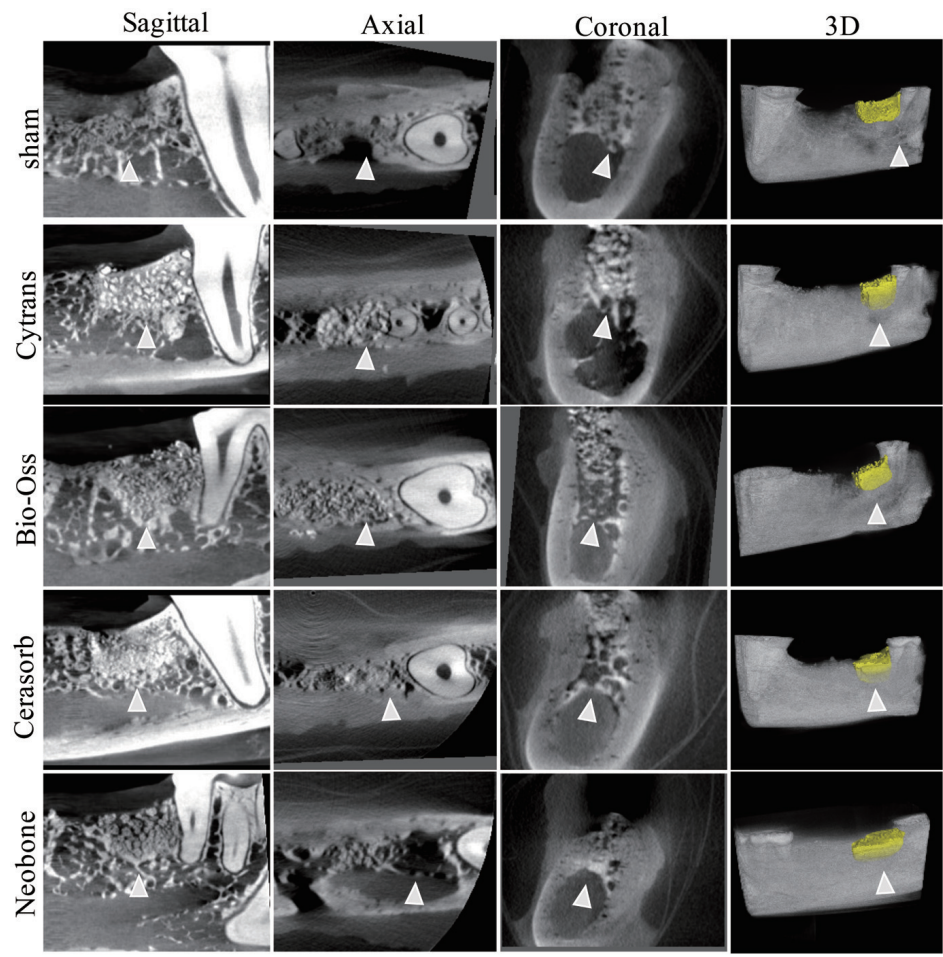

Fig. 3 Micro-CT analysis of new bone regeneration at 8 weeks post-transplantation in the animal model.

Axial, sagittal, coronal and reconstructed three-dimensional (3D) microcomputed tomography (CT) images of the defect sites of the control group (sham) and the Cytrans, Bio-Oss, Cerasorb and Neobone groups at 8 weeks posttransplantation. 
(a)

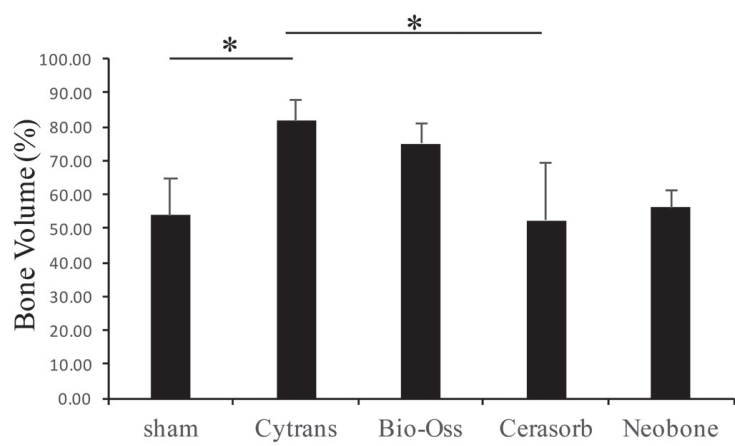

(b)

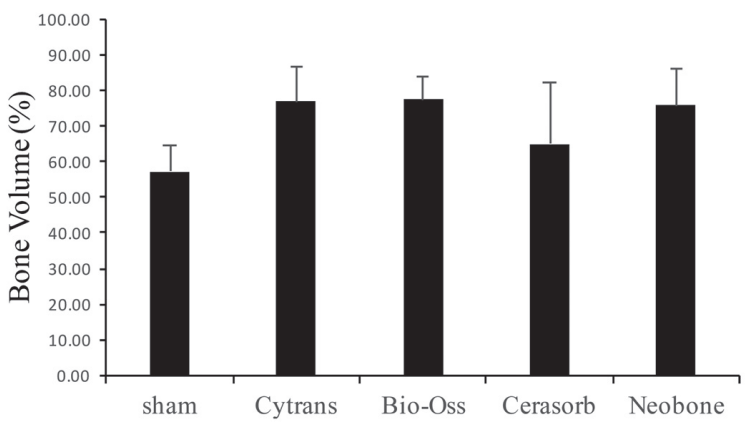

Fig. 4 Quantitative micro-CT analysis of new bone volumes.

The percentage increases in new bone volumes in the five animal groups at 4 weeks (a) and 8 weeks (b) at the sites transplanted with Cytrans, BioOss, Cerasorb and Neobone or the sham (control) treated sites. Data are expressed as the mean $\pm \mathrm{SD}$. ${ }^{*} p<0.05$ compared with the control.

differences between the five groups (sham, Cytrans, Bio-Oss, Cerasorb and Neobone) in the bone volume percentage of the newly formed hard tissue (Fig. 4b).

\section{Measurement of $A B C$ - $C E J$}

We next measured the ABC-CEJ to investigate the healing ability of the periodontal tissue post-grafting, including the cementum, periodontal ligament and alveolar bone adjacent to the tooth root. The results indicated that Cytrans, Bio-Oss and Cerasorb were slightly decreased compared with the sham group, but statistical analysis indicated that no significant differences were observed between Cytrans, Bio-Oss, Cerasorb and Neobone (Figs. 5a, b).

\section{In vivo bone regeneration ability of grafted biomaterials} by histological evaluation

We performed a histological evaluation of new bone tissue formation in the defect areas at 4 and 8 weeks. The experimental areas were sectioned and stained by hematoxylin and eosin (Fig. 6). The higher-magnification (40x) images (Figs. 6f-j, p-t) obtained from the defect area correspond to the framed regions of the lowermagnification (2.5×) images (Figs. $6 \mathrm{a}-\mathrm{e}, \mathrm{k}-\mathrm{o}$ ) at both 4 and 8 weeks post-grafting. In the sham group at lower (a)

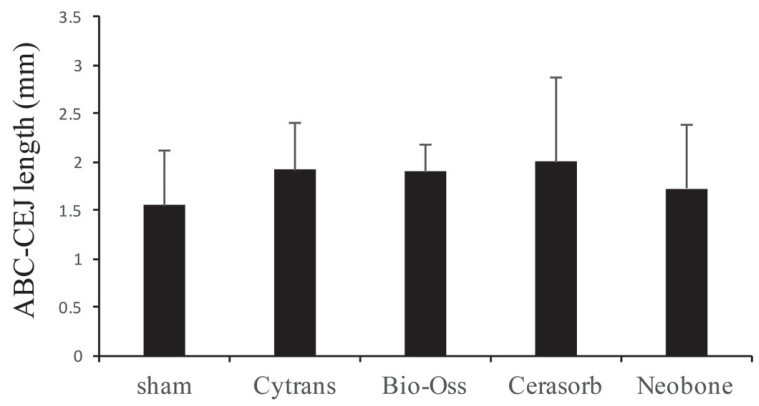

(b)

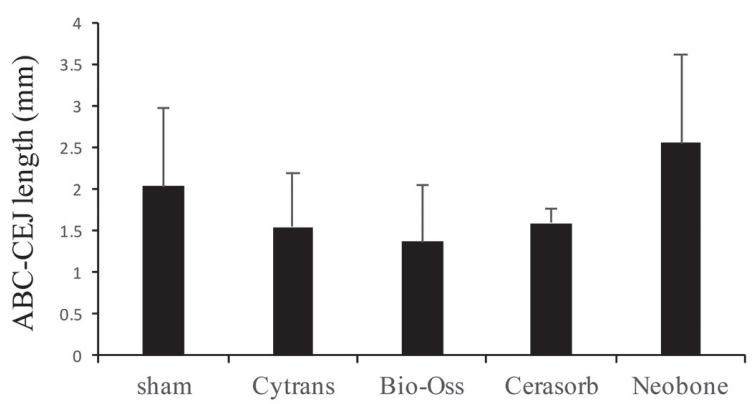

Fig. 5 Quantitativeanalysis of thecement-enameljunction to the alveolar bone crest in the transplants.

Linear bone regeneration between the cementenamel junction and alveolar bone crest (ABC-CEJ) measured along the adjacent root surface of the defect area at 4 (a) and 8 (b) weeks post-implantation. No significant differences were observed between any of the groups at either of these timepoints.

magnification, we observed minimal new bone formation (Figs. 6a, k), with most of the defect area replaced by connective tissue, which was clearly evident at highermagnification (Figs. 6f, p) at 4 and 8 weeks. In the Cytrans group, most of the grafted material was replaced with new osteoid tissue in the center and edge of the defect at both 4 (Fig. 6b) and 8 weeks (Fig. 6l). A greater amount of new bone formation was seen in the Cytrans group compared to all other groups. At higher magnification, the new bone that had formed in the Cytrans group had the typical appearance of mature bone with osteocytes integrated into the bone matrix at both 4 (Fig. 6g) and 8 weeks (Fig. 6q). The arrowheads in the images (Figs. $6 \mathrm{~g}, \mathrm{q})$ indicate the osteocytes inside the matrix. In some samples, particularly from the Bio-Oss group, most of the grated material had been retained in the defect area (Figs. 6c, m), with minimal new bone formation (Figs. $6 \mathrm{~h}, \mathrm{r})$ at both post-transplant timepoints. Compared to the Bio-Oss group, Cerasorb samples demonstrated a higher level of new bone tissue (Figs. 6d, n), which was clearly evident at higher magnification (Figs. 6i, s). The new osteoid tissue formed in the Cerasorb group at both 4 and 8 weeks was irregular, with less well organized trabeculae, a condition that is characteristic of immature bone (Figs. 6d, n). The Neobone samples 

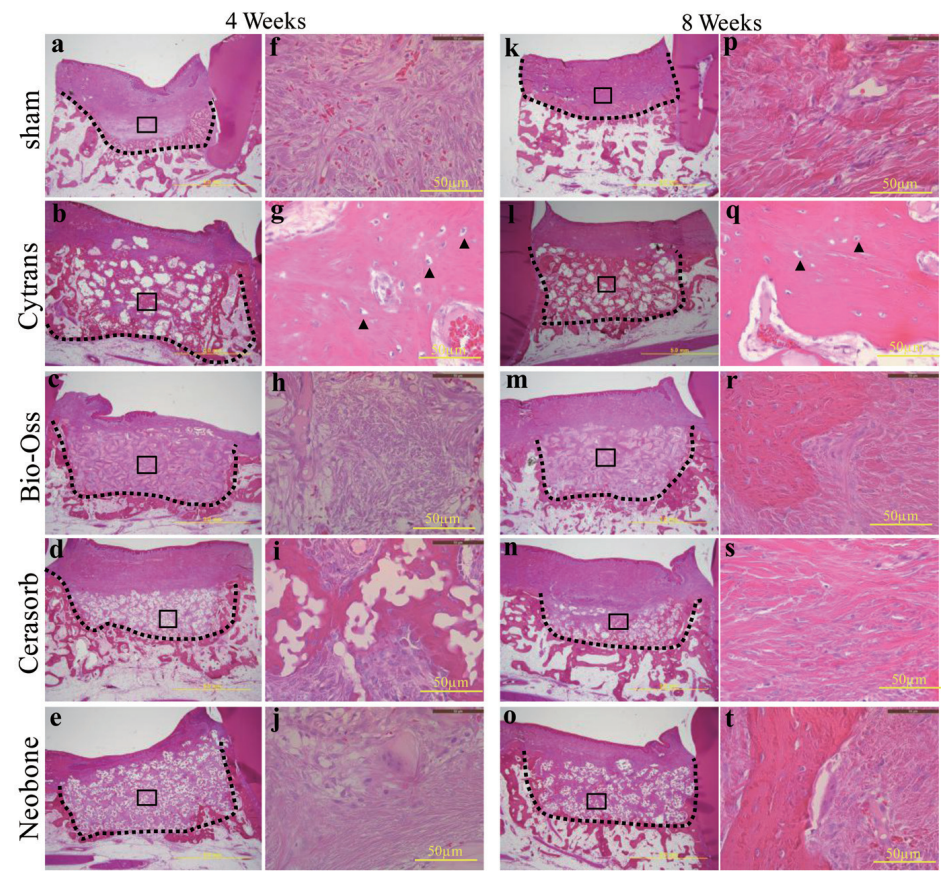

Fig. 6 Histology of new bone formation in the defect area evaluated by HE staining.

Representative images that span the area of intact bone to the area of the defect are shown. Sections were stained with hematoxylin and eosin (HE). The dotted line represents the intersection between the intact bone and the defect area for each group at $4(\mathrm{a}-\mathrm{e})$ and 8 weeks $(\mathrm{k}-\mathrm{o})$ post-transplantation. The boxed areas in $(\mathrm{a}-\mathrm{e})$ and $(\mathrm{k}-\mathrm{o})$ correspond to the higher magnification images in $(\mathrm{f}-\mathrm{j})$ and $(\mathrm{p}-\mathrm{t})$, respectively. Scale bar, $(\mathrm{a}-\mathrm{e}, \mathrm{k}-\mathrm{o}) 5 \mathrm{~mm}$.

showed a somewhat similar pattern of new osteoid tissue formation to the Cerasorb group at lower magnification (Figs. 6e, o). The new bone formed in the Neobone group showed greater cellularity however with less organized bone (Figs. 6j, t).

We quantitatively evaluated the regenerated new bone in the defect areas at 4 and 8 weeks post-implantation using photomicrographs of HE-stained sections. We made a total of nine serial HE-stained sections of the defect area of each group. From each section, we randomly selected three field areas under a microscope and obtained a total of 27 photomicrographs. Each image was analyzed for darkly stained pink areas, representing regenerated new bone, using ImageJ software to obtain quantitative data. From these results, we found that the new bone areas were significantly larger (20-25\%) in the Cytrans groups than in the sham or Bio-Oss groups at 4 weeks post-implantation. However, there was no significant difference in this regard between the Cytrans, Cerasorb and Neobone groups at 4 weeks post-implantation (Fig. $7 \mathrm{a})$. In contrast, at 8 weeks post-implantation, the Cytrans group demonstrated a 20\% larger new bone area than the sham group, which was significant, but no differences compared to the other groups (Fig. 7b). We then performed Azan staining to further evaluate new collagen fiber and bone tissue formation in the defect areas at both post-grafting timepoints (Fig. 8).

New osteoid formation indicated by positive Azan staining in Cytrans, Bio-Oss, Cerasorb and Neobone (a)

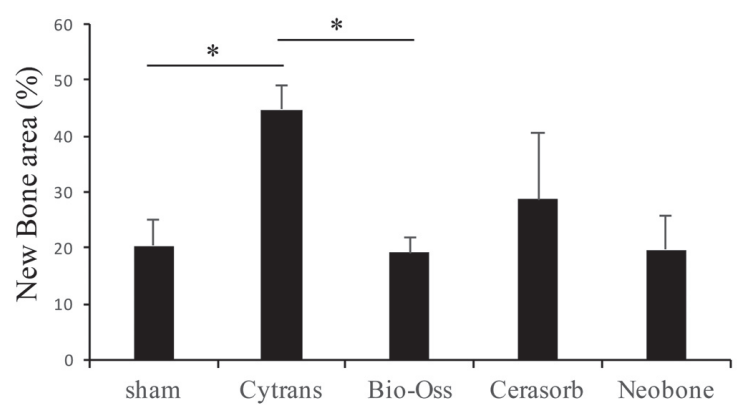

(b)

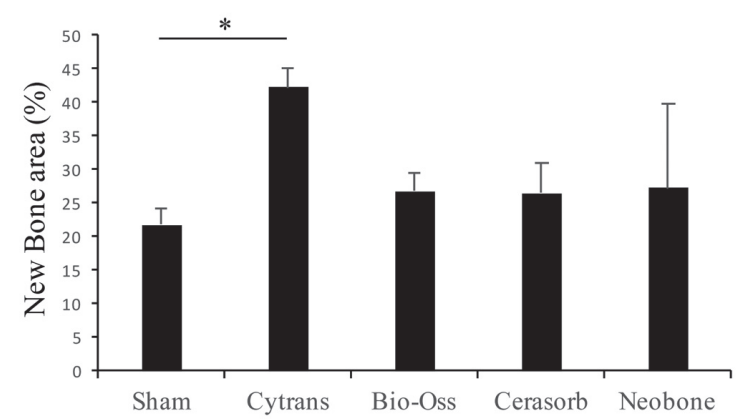

Fig. 7 Quantitative histomorphometry analysis of the new bone areas.

The percentage increases in the new bone volumes between the five study groups at 4 weeks (a) and 8 weeks (b) at the sites transplanted with Cytrans, Bio-Oss, Cerasorb and Neobone, and the sham treated animals, are shown. Data are expressed as the mean \pm SD. ${ }^{*} p<0.05$ compared with the control. 


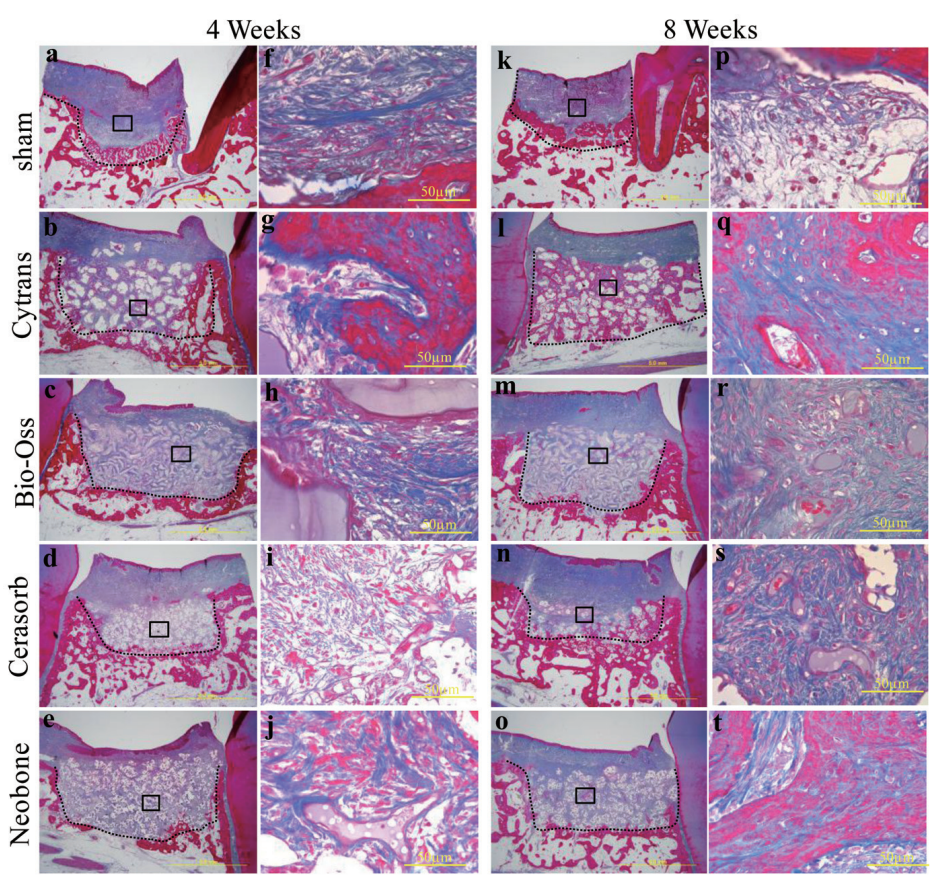

Fig. 8 Histology of collagen fiber and new bone formation in the defect areas evaluated by Azan staining.

Representative images that span the area of intact bone to the area of the defect are shown. Sections were analyzed by Azan staining. The dotted line represents the intersection between the intact bone and the defect area for each group at $4(\mathrm{a}-\mathrm{e})$ and 8 weeks $(\mathrm{k}-\mathrm{o})$. The boxed areas in $(\mathrm{a}-\mathrm{e})$ and $(\mathrm{k}-\mathrm{o})$ correspond to the higher magnification images in $(\mathrm{f}-\mathrm{j})$ and $(\mathrm{p}-\mathrm{t})$, respectively. Scale bar, $(\mathrm{a}-\mathrm{e}, \mathrm{k}-\mathrm{o}) 5 \mathrm{~mm}$.

was similar to that of HE staining. However, the Azan staining results revealed greater amount of collagen fiber formation in the Cytrans animals compared to the other groups. In the sham group at lower magnification, most of the defect area was filled with collagen fiber formation (Figs. 8a, k) at 4 and 8 weeks. At higher magnification in these controls however, no mineralized matrix with abundant connective tissue formation was observed (Figs. 8f, p) in the defect area at 4 and 8 weeks. In contrast, the Cytrans group demonstrated a mixture of collagen fiber formation and mineralized matrix at 4 weeks (Fig. 8b), and mainly mineralized bone matrix at 8 weeks (Figs. 81), indicating the maturation of bone tissue. This was confirmed at higher magnification (Figs. $8 g, q)$, where we observed that most of the defect area was filled with matured bone matrix, with integrated osteocytes and collagen fiber formation in some parts. In the Bio-Oss group, the defect area showed retained graft material (Figs. 8c, m) at both 4 and 8 weeks, similar to the $\mathrm{HE}$ staining results. In the higher magnification images in this group (Figs. 8h, r), we observed leftover graft material, a small number of dispersed erythrocytes and greater collagen fiber formation in the defect area at both 4 and 8 weeks. In the Cerasorb group in the lower magnification images (Figs. 8d, n), the defect area was filled with mostly fibrotic, collagenous tissue at 4 weeks post-transplantation. Higher magnification analysis of the Cerasorb group revealed abundant erythrocytes and evenly dispersed collagen fibers at 4 weeks (Fig. 8i), and dense collagenous tissue with dispersed cells at 8 weeks (Fig. 8s) post-implantation. In the Neobone group, the defect area was filled partially by bone-like tissue and collagenous fiber (Figs. 8e, o) at 4 and 8 weeks. At higher magnification in this group, the images revealed irregularly formed bone tissue surrounded by dense collagenous fibrous tissue (Figs. 8j, t).

Prevalence of cathepsin K-positive cells in the defect areas To evaluate the active remodeling process in the defect areas of the animal groups after implantation, we performed immunohistochemical analysis of an osteoclastic marker, cathepsin K. At 4 weeks, the Cytrans and Neobone groups demonstrated a higher number of cathepsin K-positive cells compared with the sham, BioOss, Cerasorb and Neobone groups (Figs. 9a-e, black arrow). The intensity of staining was also higher in the Cytrans group (Fig. 9b) compared to the other groups (Figs. 9a, c-e). At 8 weeks, all of the experimental groups had cathepsin K-positive cells apart from the sham animals, and these cells were located at the interface of the host tissue and regenerated new bone (Figs. 9g-j). These cathepsin K-positive cells were the multinucleated osteoclasts cells seen at the interface of the regenerated new bone and host tissue, indicative of an active remodeling process.

Prevalence of CD31-positive cells in the defect areas We performed immunohistostaining for CD31 in the 


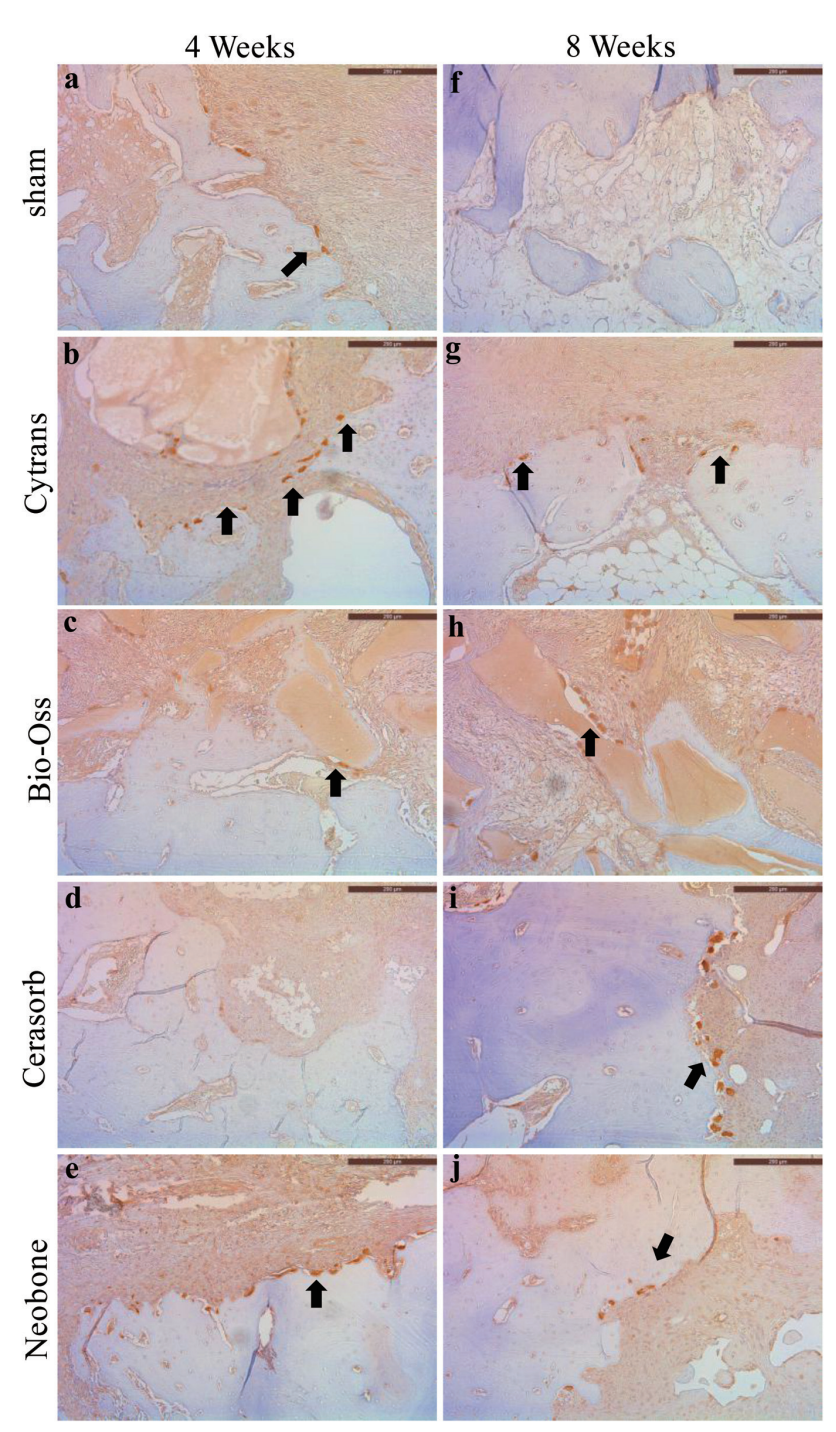

Fig. 9 Immunohistochemical analysis of the defect areas with anti-cathepsin $\mathrm{K}$ antibody.

Immunostaining of cathepsin K protein distribution in decalcified bone sections of the defect area at $4(\mathrm{a}-\mathrm{e})$ and $8(\mathrm{f}-\mathrm{j})$ weeks post-implantation. Black arrows denote a cathepsin K-positive, multinucleated osteoclast residing within a resorption pit in the region of interest. Scale bar, $200 \mu \mathrm{m}$

defect areas to evaluate the extent of neo-angiogenesis in the regenerated new bone (Fig. 10). CD31-positive blood vessels were observed in the defect area of all four implanted groups at 4 (Figs. 10a-e) and 8 weeks (Figs. $10 \mathrm{f}-\mathrm{j}$ ) into the healing period. In the Cytrans group, we observed distinct CD31-positive blood vessels (black arrow) that had penetrated the regenerated new bone at both 4 and 8 weeks. In all other groups, we observed smaller CD31-positive blood vessels in the defect area at both 4 and 8 weeks after implantation.

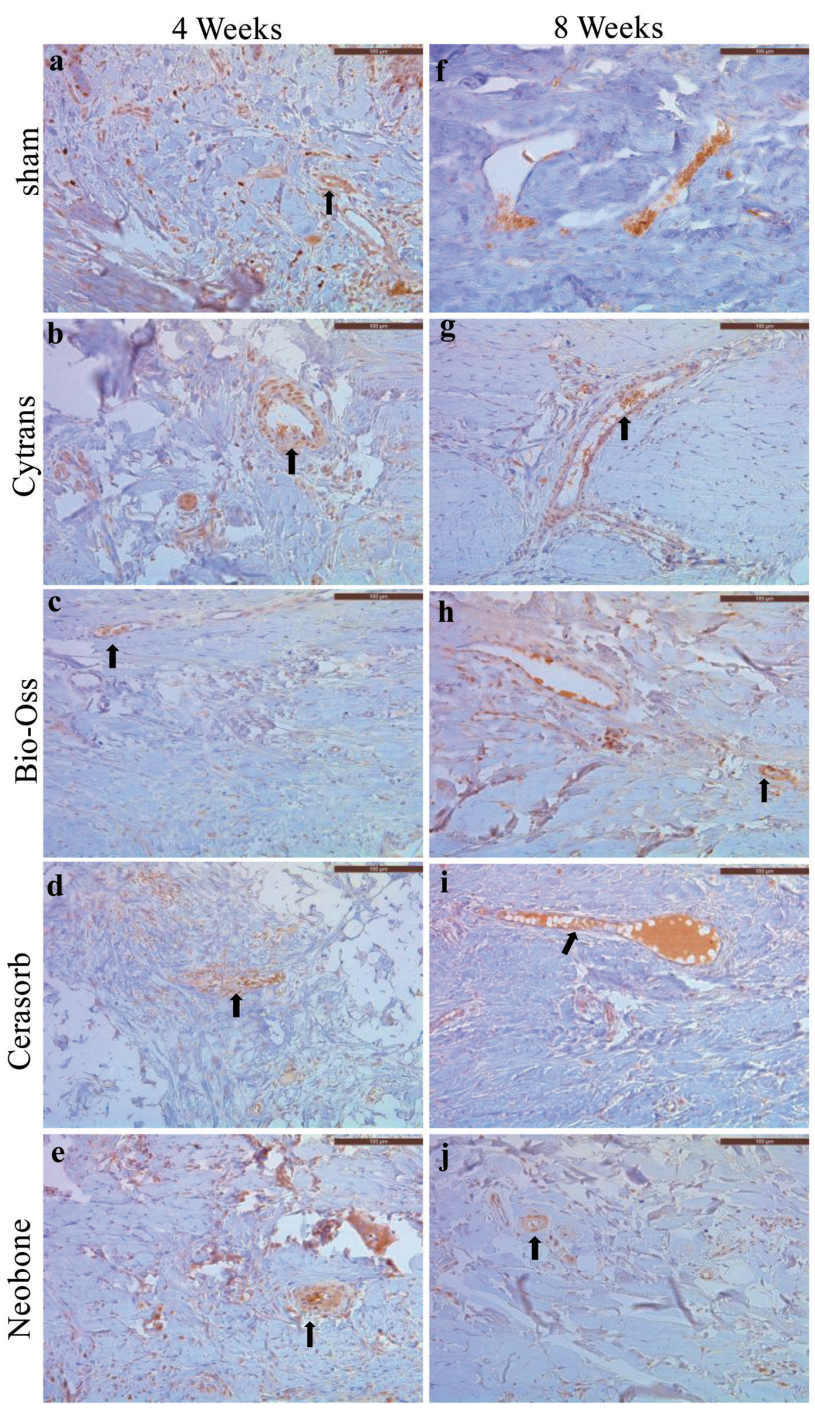

Fig. 10 Immunohistochemical analysis of CD31 expression in the defect areas.

Immunostaining of the decalcified bone sections of the defect areas with CD31 antibodies was done at $4(\mathrm{a}-\mathrm{e})$ and 8 (f-j) weeks post-implantation. Black arrows indicate CD31-positive blood vessels within the defect area of the regenerated new bone.

\section{DISCUSSION}

We have here compared the vertical bone defect healing ability of Cytrans, Bio-Oss, Cerasorb, and Neobone using a 3-wall bone defect animal model system. Although no changes to the wound healing ability of periodontal ligament, cementum or alveolar bone adjacent to the tooth root were observed, Cytrans showed an advantage over the other materials in the correction of vertical bone defects. Histological analysis revealed that all of the tested materials promoted bone healing with angiogenesis and osteoclast recruitment on the graft 
surface. Notably however, Cytrans produced faster bone healing than Bio-Oss, Cerasorb, or Neobone.

In our present analyses, faster bone healing was observed in the Cytrans group in the 3-wall vertical bone defect model compared with Bio-Oss, Cerasorb and Neobone. Our data also indicated that Cytrans promotes osteoclast recruitment, angiogenesis and osteoblast differentiation in the early stages after transplantation, likely due to the effects of carbonate and calcium. These results are consistent with previous findings and provide evidence that Cytrans is an effective bone substitute material for the treatment of vertical bone defects ${ }^{6}$.

Our animal model indicated that Cytrans, Bio-Oss, Cerasorb and Neobone produce similar bone healing processes, but that some differences exist. Nagai et al. reported previously that hBMCs incubated on $\mathrm{CO}_{3} \mathrm{Ap}$ demonstrate a higher expression of osteoblastic differentiation markers than those incubated with HA, such as type I collagen, alkaline phosphatase, osteopontin and osteocalcin ${ }^{11}$. The enhanced osteogenic ability of $\mathrm{CO}_{3} \mathrm{Ap}$ is caused by released calcium ions which can bind the cell membrane via $\mathrm{G}$ protein-coupled receptors to activate the Erk pathway ${ }^{12-14)}$. Consistently, a large amount of new bone formation has been observed following the transplantation of Cytrans in a femur defect model $^{15)}$. In our present study, Cytrans showed faster new bone formation in a 3-wall bone defect compared with Bio-Oss, Neobone and Cerasorb, based on Azan staining at 4 and 8 weeks post-implantation. Bio-Oss is composed of low-absorbable demineralized bovine bone and is one of the most clinically studied xenografts due to its expected biocompatibility and new bone formation properties ${ }^{16)}$. The morphology of Bio-Oss, which incorporate a $75-80 \%$ porosity, facilitates the migration of endothelial cells and osteoblasts, thereby creating a similar structure to human bone. Previous reports have indicated that the number of osteoblasts and osteocytes was increased 9 years after a paranasal elevation using Bio-Oss ${ }^{17)}$, but that bone replacement takes a long time with this material compared with $\beta$-TCP or $\mathrm{CO}_{3} \mathrm{Ap}^{18,19)}$. Similar to Bio-Oss, Neobone has demonstrated slow resorption because it contains no micropores in the macropore wall, and this structure provides a suitable environment for cell adhesion, matrix synthesis and calcification during bone healing ${ }^{20)}$. Previous Neobone transplantation experiments using a 4 -wall bone defect dog model have indicated that bone healing occurs at the interface of the host bone ${ }^{21}$. Cerasorb is a reconstructive bone substitute having a porous granular structure with a total porosity of approximately $65 \%$, and is absorbed by a combination of hydrolysis and cell degradation processes ${ }^{22)}$. The effectiveness of Bio-Oss, Neobone and Cerasorb in promoting bone healing have thus been already described. However, our data indicate that Cytrans is a novel and effective alternative to these materials. Furthermore, the infiltration of inflammatory cells was not observed throughout the transplantation period with Cytrans, and absorption of the graft material was observed along with the increase in new bone.

Bone healing requires bone remodeling mediated by coupling signals that are transmitted by osteoclasts to osteoblasts and drive the transition from bone resorption to formation ${ }^{23)}$. Hence, effective bone substitute grafting requires a microenvironment that activates osteoblasts and osteoclasts to reproduce remodeling at the transplantation site. In a prior transplantation assay of $\beta$-TCP, osteoclasts were reported to appear prior to newborn bone formation ${ }^{24)}$, suggesting that osteoclast activation via a scaffold is important for the healing processes promoted by bone substitute material. In our current analyses, Cytrans produced faster bone healing with earlier osteoclast appearance compared with BioOss, Neobone and Cerasorb. Since $\mathrm{CO}_{3} \mathrm{Ap}$ has increased solubility in weakly acidic environments, our results strongly suggest that carbonate release from Cytrans promotes early osteoclast recruitment and thereby lead to faster bone healing in our 3-bone wall defect model compared with the other material. These data suggest therefore that Cytrans accelerates bone healing through the initiation of bone remodeling via osteoclast activation.

Autologous bone grafting is considered to be the gold standard for correcting bone loss as it induces angiogenesis to ensure blood flow at the transplant site, thereby enabling an ample nutritional supply that improves the engraftment rate of transplants. Angiogenesis is therefore an essential event in the early stages of bone regeneration, followed by the activation of osteoclasts and osteoblasts ${ }^{23)}$. Osteogenesis requires angiogenesis, as revealed by the differentiation of mesenchymal stem cells into vascular endothelial cells via the actions of vascular endothelial growth factor secreted from multiple cells in the vasculature ${ }^{25,26}$. Previous studies in which $\beta$-TCP was transplanted into the back muscles of dogs have found that angiogenesis requires the healing of bone defects because osteoblasts and vascular endothelial cell precursors are observed near to the blood vessels during the endochondral ossification process ${ }^{27)}$. In our present study, CD31-positive endothelial cells were observed in the Cytrans material, maybe due to the blood flow and the abundant supply of nutrients that result from angiogenic processes. These findings strongly suggest that the faster bone formation we observed in the Cytrans transplants was due to the promotion of angiogenesis.

\section{CONCLUSION}

Cytrans is an effective and suitable bone substitute material for faster bone healing, that is accompanied by recruitment of osteoclasts and endothelial cells, as compared with Bio-Oss, Neobone and Cerasorb. Our current data indicate that Cytrans is a reliable bone substitute material for the treatment of vertical bone defects caused by periodontitis.

\section{ACKNOWLEDGMENTS}

We greatly thank Ms. Yayoi AOYAMA for immunohistological work. This work was supported 
by Japan Society for the Promotion of Science (JSPS) KAKENHI, Grant Number 18H02975 and 18K09592.

\section{REFERENCES}

1) Yildirim M, Spiekermann H, Biesterfeld S, Edelhoff D. Maxillary sinus augmentation using xenogenic bone substitute material Bio-Oss in combination with venous blood. A histologic and histomorphometric study in humans. Clin Oral Implants Res 2000; 11: 217-229.

2) Schlegel KA, Fichtner G, Schultze-Mosgau S, Wiltfang J. Histologic findings in sinus augmentation with autogenous bone chips versus a bovine bone substitute. Int $\mathrm{J}$ Oral Maxillofac Implants 2003; 18: 53-58.

3) Artzi Z, Tal H, Dayan D. Porous bovine bone mineral in healing of human extraction sockets: 2. Histochemical observations at 9 months. J Periodontol 2001; 72: 152-159.

4) Merkx MA, Maltha JC, Stoelinga PJ. Assessment of the value of anorganic bone additives in sinus floor augmentation: a review of clinical reports. Int J Oral Maxillofac Surg 2003; 32: 1-6.

5) Fujita R, Yokoyama A, Kawasaki T, Kohgo T. Bone augmentation osteogenesis using hydroxyapatite and betatricalcium phosphate blocks. J Oral Maxillofac Surg 2003; 61: 1045-1053.

6) Kokubo T, Kim HM, Kawashita M. Novel bioactive materials with different mechanical properties. Biomaterials 2003; 24: 2161-2175.

7) Matsuya S, Lin X, Udoh K, Nakagawa M, Shimogoryo R, Terada Y, et al. Fabrication of porous low crystalline calcite block by carbonation of calcium hydroxide compact. J Mater Sci Mater Med 2007; 18: 1361-1367.

8) Fujisawa K, Akita K, Fukuda N, Kamada K, Kudoh T, Ohe G, et al. Compositional and histological comparison of carbonate apatite fabricated by dissolution-precipitation reaction and Bio-Oss ${ }^{\circledast}$. J Mater Sci Mater Med 2018; 29: 121.

9) Ayukawa Y, Suzuki Y, Tsuru K, Koyano K, Ishikawa K. Histological comparison in rats between carbonate apatite fabricated from gypsum and sintered hydroxyapatite on bone remodeling. Biomed Res Int 2015; 2015: 579541.

10) Kudoh K, Fukuda N, Kasugai S, Tachikawa N, Koyano K, Matsushita Y, et al. Maxillary sinus floor augmentation using low-crystalline carbonate apatite granules with simultaneous implant installation: First-in-human clinical trial. J Oral Maxillofac Surg 2019; 77: 985.

11) Nagai H, Kobayashi-Fujioka M, Fujisawa K, Ohe G, Takamaru $\mathrm{N}$, Hara K, et al. Effects of low crystalline carbonate apatite on proliferation and osteoblastic differentiation of human bone marrow cells. J Mater Sci Mater Med 2015; 26: 99.

12) Seol YJ, Park JY, Jung JW, Jang J, Girdhari R, Kim SW, et al. Improvement of bone regeneration capability of ceramic scaffolds by accelerated release of their calcium ions. Tissue Eng Part A 2014; 20: 2840-2849.

13) Ha SW, Park J, Habib MM, Beck GR Jr. Nano-hydroxyapatite stimulation of gene expression requires Fgf receptor, phosphate transporter, and Erk1/2 signaling. ACS Appl Mater Interfaces 2017; 9: 39185-39196.

14) Hofer AM, Lefkimmiatis K. Extracellular calcium and cAMP: second messengers as "third messengers"? Physiology (Bethesda) 2007; 22: 320-327.

15) Takeuchi H, Nagayama $M$, Imaizumi $Y$, Tsukahara $T$, Nakazawa J, Kusaka Y, et al. Immunohistochemical analysis of osteoconductivity of beta-tricalcium phosphate and carbonate apatite applied in femoral and parietal bone defects in rats. Dent Mater J 2009; 28: 595-601.

16) Orsini G, Traini T, Scarano A, Degidi M, Perrotti V, Piccirilli $\mathrm{M}$, et al. Maxillary sinus augmentation with Bio-Oss particles: a light, scanning, and transmission electron microscopy study in man. J Biomed Mater Res B Appl Biomater 2005; 74: 448457.

17) Traini T, Valentini P, Iezzi G, Piattelli A. A histologic and histomorphometric evaluation of anorganic bovine bone retrieved 9 years after a sinus augmentation procedure. J Periodontol 2007; 78: 955-961.

18) Berglundh T, Lindhe J. Healing around implants placed in bone defects treated with Bio-Oss. An experimental study in the dog. Clin Oral Implants Res 1997; 8: 117-124.

19) Shin HI, Sohn DS. A method of sealing perforated sinus membrane and histologic finding of bone substitutes: a case report. Implant Dent 2005; 14: 328-333.

20) de Groot K. Bioceramics consisting of calcium phosphate salts. Biomaterials 1980; 1: 47-50.

21) Ishikawa K, Miyamoto Y, Tsuchiya A, Hayashi K, Tsuru K, Ohe G. Physical and histological comparison of hydroxyapatite, carbonate apatite, and beta-tricalcium phosphate bone substitutes. Materials (Basel) 2018; 11: 1993.

22) Wiltfang J, Merten HA, Schlegel KA, Schultze-Mosgau S, Kloss FR, Rupprecht S, et al. Degradation characteristics of alpha and beta tri-calcium-phosphate (TCP) in minipigs. J Biomed Mater Res 2002; 63: 115-121.

23) El-Jawhari JJ, Jones E, Giannoudis PV. The roles of immune cells in bone healing; what we know, do not know and future perspectives. Injury 2016; 47: 2399-2406.

24) Kondo N, Ogose A, Tokunaga K, Ito T, Arai K, Kudo N, et al. Bone formation and resorption of highly purified betatricalcium phosphate in the rat femoral condyle. Biomaterials 2005; 26: 5600-5608.

25) Hielscher D, Kaebisch C, Braun BJV, Gray K, Tobiasch E. Stem cell sources and graft material for vascular tissue engineering. Stem Cell Rev 2018; 14: 642-667.

26) Luo C, Yang X, Li M, Huang H, Kang Q, Zhang X, et al. A novel strategy for in vivo angiogenesis and osteogenesis: magnetic micro-movement in a bone scaffold. Artif Cells Nanomed Biotechnol 2018; 46: 636-645.

27) Kondo N, Ogose A, Tokunaga K, Umezu H, Arai K, Kudo N, et al. Osteoinduction with highly purified beta-tricalcium phosphate in dog dorsal muscles and the proliferation of osteoclasts before heterotopic bone formation. Biomaterials 2006; 27: 4419-4427. 